\section{Novel Strategy for Colorectal Liver Metastases -Estimated by the Concept for Hepatocyte Growth Factor}

\section{Abstract}

Colorectal cancer is the third most common epithelial malignancy worldwide. At some point in the natural course of patients with this disease, up to $50 \%$ will develop metastasis to the liver, i.e., colorectal liver metastasis (CLM), which represents one of the most common threats to life. A variety of therapeutic approaches to treat this problematic disease have been attempted that have now resulted in the acceptance of an aggressive strategy even for advanced cases. However, the surgical indications for resection of synchronous metastases and the optimal timing of hepatectomy are still controversial and widely debated. In the present review, chemotherapeutic strategies involving recently developed regimes and/or hepatectomy are proposed based not only on the clinical data but also on biological concepts in which chemotherapy causes hepatocyte growth factor (HGF)-related epithelial mesenchymal transition (EMT).

Keywords: Colorectal cancer, Epithelial malignancy; Hepatectomy

Received: October 28, 2015, Accepted: November 18, 2015, Published: November 26, 2015

\section{Introduction}

Colorectal cancer is the third most common epithelial malignancy worldwide [1], accounting for over 130,000 new cases and almost 50,000 deaths in 2015 [2]. At some point in the natural course of patients with this disease, up to $50 \%$ will develop metastasis to the liver [3], i.e., colorectal liver metastasis (CLM), which represents one of the most common threats to life. A variety of therapeutic approaches have been attempted for this problematic disease that have now resulted in the acceptance of an aggressive strategy even for advanced cases [4]. However, the surgical indications for resection of synchronous metastases and the optimal timing of hepatectomy (simultaneous or staged) are still controversial and widely debated [5]. Because simultaneous major hepatectomy is associated with severe rates of morbidity (36.1\% vs. $17.6 \%)$ and mortality (8.3\% vs. $1.4 \%)$ [6] in comparison with staged hepatectomy, a staged operation has been recommended. However, based on the results of similar operative times, intraoperative blood loss and complication rates [4], simultaneous resection appear to offer enhanced safety $[7,8]$. Thus, simultaneous treatments have been evaluated as grade

\author{
Shinji Osada ${ }^{1}$, \\ Satoshi Matsui ${ }^{1}$, \\ Yoshiyuki Sasaki², \\ Hisashi llmaí2, \\ Yoshihiro Tanaka², \\ Nobuhisa Matsuhashi², \\ Naoki Okumura ${ }^{2}$, \\ Kazuhiro Yoshida
}

\section{Multidisciplinary Therapy for Hepato-Biliary-Pancreatic Cancer, Gifu University School of Medicine, Japan \\ 2 Surgical Oncology, Gifu University School of Medicine, Japan}

Corresponding author: Shinji Osada MD

” sting@gifu-u.ac.jp

Multidisciplinary Therapy for Hepato-BiliaryPancreatic Cancer, Gifu University School of Medicine, 1-1 Yanagido Gifu city, 501-1194, Japan

Tel: $+81-58230-6233$

Fax: $+81-58230-1074$

Citation: Osada S (2015) Novel Strategy for Colorectal Liver Metastases -Estimated by the Concept for Hepatocyte Growth Factor. Arch Cancer Res 1: 1.

C under the recommended guidelines [9], but no randomized trials have been undertaken to confirm this. Except these safety concepts for such surgical procedures, oncological approach should be critical.

Recently developed chemotherapy of 5-fluorouracil (5FU)/ folinic acid with irinotecan (FOLFIRI) or oxaliplatin (FOLFOX) has been found to be beneficial not only for initially unresectable but also even resectable synchronous metastases [10]. Because a prospective phase II study of these treatment regimens demonstrated a response rate of $66 \%$ [11] and a maximum resection rate of $82 \%$ [12], the past data indicate that traditional chemotherapeutic regimens are decreasingly being used. When arguing the timing of hepatectomy and whether it should be 
performed first or be staged, the contents and results from the most recent chemotherapeutic developments should be considered [13]. In the condition in which only $15-20 \%$ of patients are initial candidates for resection [14], the goals of chemotherapy might include conversion of cases from unresectable and identification of the best candidates for a curative treatment. In the present review, chemotherapeutic strategies involving FOLFOX or FOLFIRI and/or hepatectomy will be proposed based not only on the available clinical data but also on biological concepts.

\section{Determination of Resectability}

\section{Combination of surgical procedure with chemotherapy}

The median survival time (MST) of patients undergoing chemotherapy for the treatment of CLM was reported to increase from 22.2 months to 49.8 months with the addition of hepatectomy [15], indicating that its combination is very important. The survival rate of patients with unresectable CLM is quite poor, under $2 \%$ at 5 years, but in patients converted to resection, it is clearly improved, $33 \%$ at 5 years and $27 \%$ at 10 years [16]. Conversion is one of the most important factors arguing for prognosis in patient with CLM. From observations of unresectable CLM, the usual response rate to FOLFOX or FOLFIRI was reported to be around 50\% [17]. Despite the risk of worse liver function [18], chemotherapy itself is focused not only on improving patient prognosis [19] but also on converting inoperable cases to resectable cases [20]. The resection rate also correlates with the chemotherapy-induced response rate [17]. Actually, for patients with inoperable CLM, $40 \%$ [21] or $25.7 \%$ [22] were converted to resectable cases by these recent chemotherapeutic regimes. Among these studies, surgical indications were decided by oncological factors, such as tumor number or size and distance of the free margin, or for clinical reasons such as remnant liver volume.

Non-optimal resection is defined when patients have a number of liver tumors, maximal diameter tumors, synchronous metastases, the presence of positive lymph node metastases, tumor marker levels and tumors located near blood vessels [23]. Another report described tumor diameter and the number of tumors in CLM as critical determinates of nonresection [24]. In our previous study [13], the 3-year survival rate and MST were significantly better $(p=0.0127)$ for patients with two or fewer tumors than for patients with three or more tumors. Furthermore, in patients in whom synchronous liver tumors were detected, the 3-year survival rate and MST after staged hepatectomy were significantly better than those after simultaneous hepatectomy $(p=0.0467)$, and the MST for patients with two or fewer tumors was significantly longer than that for patients with three or more tumors. It appears that tumor number might be important for patient survival, and a cut-off point set at two tumors may be reasonable for accepting primary hepatectomy. In contrast, although the surgical margin is associated with long-term prognosis [25], current reports demonstrate a negative margin to have no effect on survival, risk of recurrence or site of recurrence in the era of modern chemotherapy $[26,27]$.

\section{Correlation of C-MET with Colorectal Liver Metastases}

\section{A possible biomarker to evaluate therapeutic procedures}

Hepatocyte growth factor (HGF) and its receptor, c-Met, have been studied in the progression of colorectal cancer [28], and the c-Met pathway is indicated to play a critical role in colorectal carcinogenesis. Although liver metastasis was significantly higher in a patient group with high expression of c-Met, in the patients with CLM, immunodetection of high-grade c-Met activity in the primary tumor changed to low-grade activity in the metastatic sites [29]. An experimental mouse study also showed that expression of c-Met decreased from culture conditions to metastasis in a time- and tumor size-dependent manner [28]. Even within single tumors, there was a difference in c-Met expression whereby it was increased in the growing invasive periphery but decreased in the established central regions, indicating that its volume decreases with the increase in cell density as described previously [29]. To explain the evidence for this c-Met reduction, one concept for cancer growth and invasion has focused on epithelial mesenchymal transition (EMT), in which a cancer cell changes from being a localized type to one with invasive and/ or metastatic ability as a step in its evolution [30]. Substantial activation of the HGF/c-Met pathway also leads to scattering and invasion of cancer cells through activation of the cell signaling pathway, and it may regulate EMT [31]. Further, a high serum circulating level of HGF is related to poor prognosis of patients with small cell lung cancer through a high rate of metastasis to several organs [32]. In observations of hepatocellular carcinoma, HGF was found to directly promote not only carcinogenesis [33] but also EMT [34]. After hepatectomy as a treatment for liver metastasis, activation of the HGF/c-Met pathway occurs as liver regeneration progresses, indicating that the increased level of serum HGF might prompt the growth of c-Met-expressed colorectal cancer cells. However, it is not necessary to clarify this because c-Met expression in tumor tissue decreases at the metastatic site, as described above.

From the observation that the inhibition of c-Met expression reduces the number and size of liver metastases as shown in Figure 1 [35], cancer cells with high expression of c-Met gain motility for progression to the vessels and/or distant organs (Figure 2). The HGF/c-Met system mediates cancer progression from local expansion to sites of distant metastasis via the process of EMT.

\section{A novel concept based on C-MET expression}

The EMT-related cell signaling includes not only stem cell-like properties but also resistance to chemo/radiation therapy under the self-renewal process [36]. Namely, there is a possibility for cancer therapy through inhibition of EMT not only to reduce metastasis but also to improve drug sensitivity [37]. Long-term 


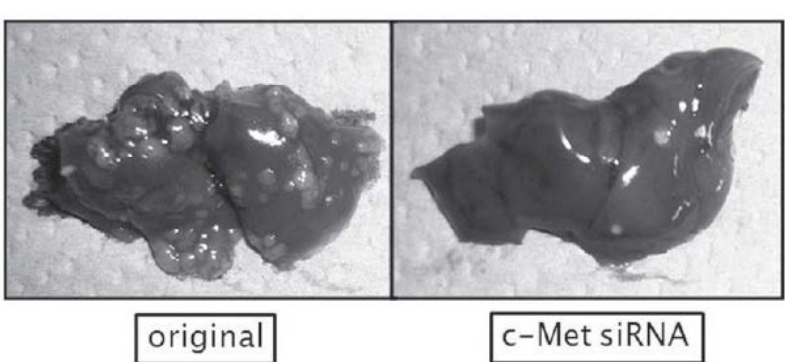

Figure 1 Effect of c-Met on liver metastasis. The protein and mRNA of c-Met were knocked down to about $30 \%$ of the control value by siRNA. On the 21st day after injection of cancer cells in the spleen, numerous metastatic tumors were detected in the liver, but cancer cells in which c-Met expression was knocked down clearly resulted in fewer tumors.

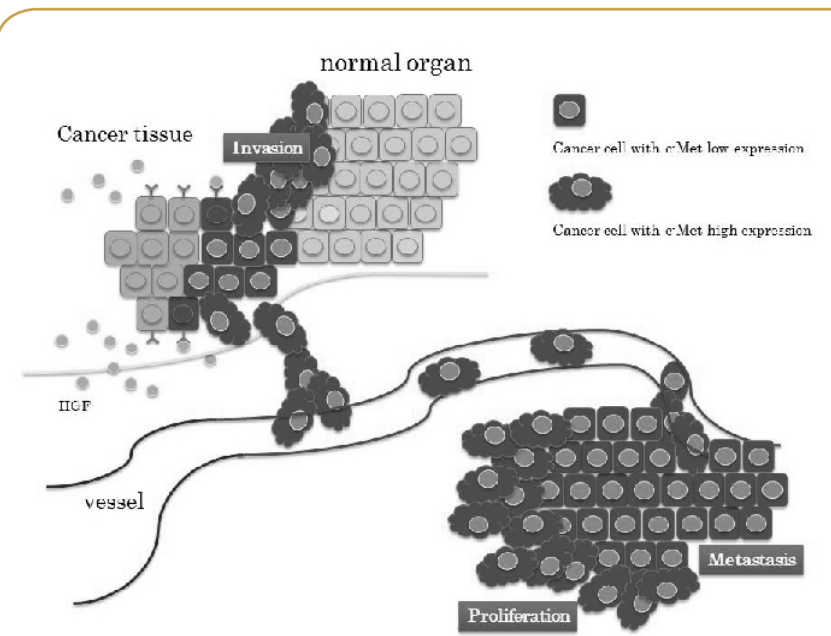

Figure 2 Cancer invasion according to the expression of c-Met. Highly expressed c-Met cancer cells gain motility for progression to the vessels and/or distant organs. At metastatic sites, there was a difference in c-Met expression whereby it was increased in the growing invasive periphery but decreased in the established central regions.

use of chemotherapeutic agents induces drug-resistant and distant metastases, and its mechanism might be correlated to activation of the signaling pathway with EMT [38,39]. In fact, paclitaxel-resistant ovarian cancer cells showed phenotypic changes consistent with EMT, with decreased expression of the factor for cell-cell contact [40]. In gefitinib-resistant lung cancer cells as well, the presence of EMT is suggested by phenotypic changes such as a spindle-cell shape and increased formation of pseudopodia [41]. Loss or low expression of cell-cell adhesionrelated factors leads to a poor patient prognosis because liver metastasis occurs more frequently [42], whereas high expression of these factors relates to higher chemosensitivity [43]. Actually, however, the early response, but not the long-term reaction, to these agents remains unclear and controversial.

5FU, which has held a central position in chemotherapy for several solid types of cancer, is a critical component in the
FOLFOX or FOLFILI regimens. It is the first rationally designed anti-metabolite to achieve its therapeutic efficacy through inhibition of DNA repair [44]. Its metabolism is regulated mainly by enzymes such as thymidylate synthase (TS) in the process of DNA synthesis [45]. Colorectal cancer patients with low levels of TS were reported to have better prognosis than those with high levels [46], indicating that the expression of TS appears to be a more useful indicator for the prediction of chemosensitivity. Our recent study showed that pretreatment with HGF induced EMTmediated higher sensitivity to 5FU through the reduction of TS by the down-regulation of cell cycle-related factors [47]. The increased expression of the $5 \mathrm{FU}$-induced cellular death signal was also detected during HGF-mediated EMT. Taken together, HGF-induced cell scattering accelerates migration to distal organs but increases the chemosensitivity to 5FU. Then, staged hepatectomy could be performed as a radical step by removing the visible solid mass after extermination of the EMT-cancer cells by neoadjuvant chemotherapy (Figure $\mathbf{3}$ ). In addition, observation of the HGF-related biological process may lead to a novel concept in which a hepatectomy-induced high serum level of HGF for liver regeneration allows drug-resistant cancer cells to become chemosensitive again through the process of EMT. To support this concept by another approach, the presence of cancer cell networks is expected. Recently, cancer cell-induced interleukin-6, CXCL-8 (interleukin-8) as a member of the chemokine family, or C-C chemokine motif ligand 2/5 was found to inhibit druginduced apoptosis and stimulate cancer cell proliferation [48]. Hepatectomy mediates not only the reduction of tumor mass but also blockage of the cancer growth network related to these types of cytokines. However, further investigation into the concept of EMT is still necessary to determine the therapeutic strategy with the use of anticancer agents.

\section{Timing of Surgery and the approach}

Determining the favorable timing of hepatectomy during chemotherapy is another critical problem. The most preferable time for surgery depends not only on its effect to impair cancer invasion but also on the negative influences caused by anticancer drugs [49]. Indeed, the complication rate of hepatectomy after certain neoadjuvant chemotherapies was suggested to increase if therapy includes more than six cycles [50]. In contrast, the most favorable outcomes with FOLFOX or FOLFIRI were detected at 9 weeks [51] or 8 weeks [52], respectively, and the chemotherapeutic effect disappears after 4 months or more [53]. The tumor shrinkage rate by chemotherapy in our patients [54] was shown to be highest at the start of therapy, but it decreased gradually after 100 days (14 or 15 weeks). Using tumor size as a response to chemotherapy, Adam et al. [55] classified the individual efficacy of chemotherapy in cases of colorectal cancer with liver metastasis into three groups: response type ( $>50 \%$ decrease in tumor size), stabilization type $(<25 \%$ increase or $<50 \%$ decrease), and progression type ( $>25 \%$ increase). The 5-year survival was much lower in the patients with progression type compared with the response and stabilization types ( $8 \%$ vs. $37 \%$ and $30 \%$, respectively; $\mathbf{p}<0.0001$ ), and disease-free survival was $3 \%$ compared with $21 \%$ and $20 \%$, respectively ( $p=0.02$ ), indicating that tumor progression before surgery is associated with a poor 


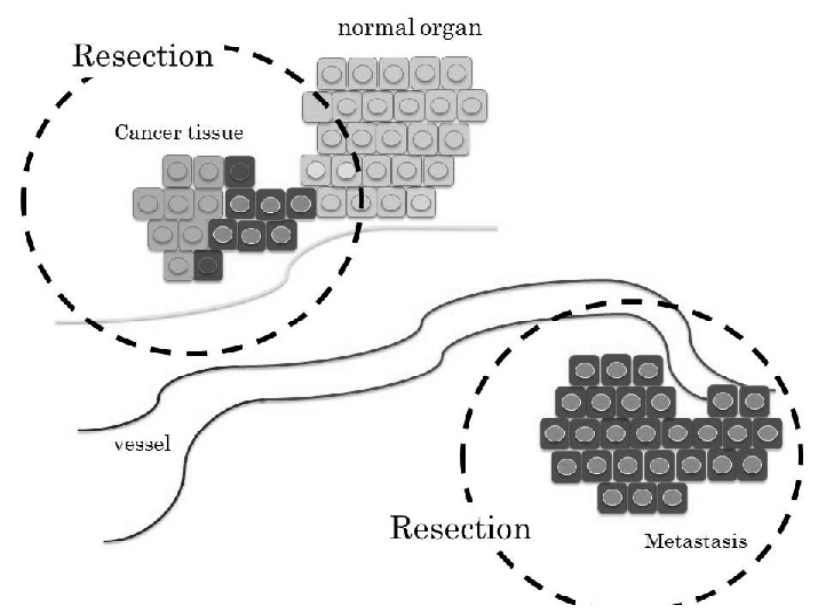

Figure 3 The effect of surgery after chemotherapy. The most favorable strategy might be a staged hepatectomy in which chemotherapy first conquers cancer cells with high expression of c-Met and then hepatectomy leads to radical therapy. outcome, even after potentially curative hepatectomy. Because the 5-year overall survival rate for neoadjuvant chemotherapy was $71 \%$ in responders and $15 \%$ in non-responders, response to chemotherapy was suggested to be a significant prognostic factor after radical hepatectomy [56]. In our study [54], even if chemotherapy was effective but was temporary in the initial short-term period, the prognosis was found to be worse. In contrast, if the shrinkage rate remained continuous at a certain level for 12 weeks after the start of chemotherapy, a planned hepatectomy was suggested to lead to significantly favorable outcomes in terms of disease-free survival. Clinical studies such as the EORTC (European Organization for Research and Treatment of Cancer)-40983 study [10] also demonstrated optimal timing of surgery to be at 12 weeks. To reduce postoperative complications,
4 weeks are necessary to wash out anticancer drugs [28]. Taken together, the optimal timing of hepatectomy might be based on the shrinkage rate at 12 weeks plus an additional 4-week rest period.

Critical factors in the determination of resection are not only number or size of the tumors but also their location. Namely, the distance of the tumor from major blood vessels is also important to predict the liver resection volume. In our study to evaluate the effect of neo-adjuvant chemotherapy with relation to vessels [57], preoperative chemotherapy achieved a tumor distance from the vessels of $10.9 \pm 7.0 \mathrm{~mm}$, and a shrinkage rate for tumor detachment from the vessels was calculated as $63.6 \pm 12.4 \%$. An adequate surgical margin in CLM is $5 \mathrm{~mm}$ for an acceptable prognosis [27], indicating that chemotherapy could increase the rate of radical operations performed. In the CELIM trial, the objective response rate with the single use of FOLFOX or FOLFIRI was $41 \%$, but it increased to $70 \%$ with the addition of a molecular targeting drug [58], indicating that the combination plays a critical role in achieving tumor separation from vessels.

\section{Novel Proposal for the Future as a Conclusion}

Management of patients with CLM is complex, and the optimal approach for timing of resection is also controversial. Chemotherapy, especially that delivered by favorable combination with molecular targeting agents, even for resectable CLM, appears to be acceptable [59]. Clinical studies have shown that neoadjuvant chemotherapy improves the prognosis even for patients with synchronous liver metastases. From the experimental approach, the presence of HGF was found to increase 5FU-induced cell death by an EMT-related cellular condition. As described here, the most favorable strategy might be a staged hepatectomy in which chemotherapy first conquers cancer cells with high expression of c-Met and then hepatectomy leads to radical therapy. The best procedure in terms of concrete timing is to plan a hepatectomy after 12 weeks of chemotherapy plus rest for 4 weeks. Clinical trials performed to understand the biological concepts will lead to the possibility of increased excellent performance of surgical resection. 


\section{References}

van Santvoort HC, Braam HJ, Spekreijse KR, Koning NR, de Bruin PC, et al. (2014) Peritoneal carcinomatosis in t4 colorectal cancer: occurrence and risk factors. Ann Surg Oncol 21: 1686-1691.

2 Siegel RL, Miller KD, Jemal A (2015) Cancer statistics, 2015 CA Cancer J Clin 65: 5-29.

3 Lochan R, White SA, Manas DM (2007) Liver resection for colorectal liver metastasis. Surg Oncol 16: 33-45.

4 Osada S, Imai H, Sasaki Y, Tanaka Y, Tokuyama Y, et al. (2012) Strategy for synchronous and multiple liver metastasis. Hepatogastroenterology 59: 198-203.

5 Brouquet A, Mortenson MM, Vauthey JN, Rodriguez-Bigas MA, Overman MJ, et al. (2010) Surgical strategies for synchronous colorectal liver metastases in 156 consecutive patients: classic, combined or reverse strategy? J Am Coll Surg 210: 934-941.

6 Reddy SK, Pawlik TM, Zorzi D, Gleisner AL, Ribero D, et al. (2007) Simultaneous resections of colorectal cancer and synchronous liver metastases: a multi-institutional analysis. Ann Surg Oncol 14: 34813491.

7 Sakamoto Y, Fujita S, Akasu T, Nara S, Esaki M, et al. (2010) Is surgical resection justified for stage IV colorectal cancer patients having bilobar hepatic metastases?--an analysis of survival of 77 patients undergoing hepatectomy. J Surg Oncol 102: 784-788.

8 Chen J, Li Q, Wang C, Zhu H, Shi Y, et al. (2011) Simultaneous vs. staged resection for synchronous colorectal liver metastases: a metaanalysis. Int J Colorectal Dis 26: 191-199.

9 Hillings $\varnothing$ JG, Wille-Jørgensen P (2009) Staged or simultaneous resection of synchronous liver metastases from colorectal cancer--a systematic review. Colorectal Dis 11: 3-10.

10 Nordlinger B, Sorbye H, Glimelius B, Poston GJ, Schlag PM, et al. (2008) Perioperative chemotherapy with FOLFOX4 and surgery versus surgery alone for resectable liver metastases from colorectal cancer (EORTC Intergroup trial 40983): a randomised controlled trial. Lancet 371: 1007-1016.

11 Falcone A, Ricci S, Brunetti I, Pfanner E, Allegrini G, et al. (2007) Phase III trial of infusional fluorouracil, leucovorin, oxaliplatin, and irinotecan (FOLFOXIRI) compared with infusional fluorouracil, leucovorin, and irinotecan (FOLFIRI) as first-line treatment for metastatic colorectal cancer: the Gruppo Oncologico Nord Ovest. J Clin Oncol 25: 1670-1676.

12 Ychou M, Viret F, Kramar A, Desseigne F, Mitry E, et al. (2008) A tritherapy with fluorouracil/leucovorin, irinotecan and oxaliplatin (FOLFILINOX): a phase II study in colorectal cancer patients with unresectable liver metastases. Cancer Chemother Pharmacol 62: 195-200.

13 Osada S, Imai H, Sasaki Y, Tanaka Y, Okumura N, et al. (2014) Surgical Indications for Multiple and Synchronous Liver Metastases from Colorectal Cancer. Hepatogastroenterology 61: 722-726.

14 de Jong MC, Pulitano C, Ribero D, Strub J, Mentha G, et al. (2009) Rates and patterns of recurrence following curative intent surgery for colorectal liver metastasis: an international multi-institutional analysis of 1669 patients. Ann Surg 250: 440-448.

15 Dexiang Z, Li R, Ye W, Haifu W, Yunshi Z, et al. (2012) Outcome of patients with colorectal liver metastasis: analysis of, 613 consecutive cases. Ann Surg Oncol 19: 2860-2868.
16 Adam R, Wicherts DA, de Haas RJ, Ciacio O, Lévi F, et al. (2009) Patients with initially unresectable colorectal liver metastases: is there a possibility of cure? J Clin Oncol 27: 1829-1835.

17 Folprecht G, Grothey A, Alberts S, Raab HR, Köhne CH (2005) Neoadjuvant treatment of unresectable colorectal liver metastases: correlation between tumour response and resection rates. Ann Oncol 16: 1311-1319.

18 Haraldsdottir S, Wu C, Bloomston M, Goldberg RM (2013) What is the optimal neo-adjuvant treatment for liver metastasis? Ther Adv Med Oncol 5: 221-234.

19 Osada S, Kanematsu M, Imai H, Goshima S, Sugiyama Y (2005) Evaluation of extracellular signal regulated kinase expression and its relation to treatment of hepatocellular carcinoma. J Am Coll Surg 201: 405-411.

20 Ribero $D$, Wang $H$, Donadon $M$, Zorzi $D$, Thomas $M B$, et al. (2007) Bevacizumab improves pathologic response and protects against hepatic injury in patients treated with oxaliplatin-based chemotherapy for colorectal liver metastases. Cancer 110: 2761 2767.21.

21 Wong R, Cunningham D, Barbachano Y, Saffery C, Valle J, et al. (2011) A multicentre study of capecitabine, oxaliplatin plus bevacizumab as perioperative treatment of patients with poor-risk colorectal liveronly metastases not selected for upfront resection. Ann Oncol 22: 2042-2048.

22 Ye LC, Liu TS, Ren L, Wei Y, Zhu DX, et al. (2013) Randomized controlled trial of cetuximab plus chemotherapy for patients with KRAS wild-type unresectable colorectal liver-limited metastases. J Clin Oncol 31: 1931-1938.

23 Nordinger B, Van Custsem E, Gruenberger T (2009) European colorectal metastases treatment group; six international colorectal liver metastases workshop. Combination of surgery and chemotherapy and the role of targeted agents in the treatment of patients with colorectal liver metastases: recommendations from an expert panel. Ann Oncol 20: 985-990.

24 GarufiC, Torsello A, TumoloS (2010) Cetuximab plus chronomodulated irinotecan, 5-fluoroacil, leucovorin and oxaliplatin as neoadjuvant chemotherapy in colorectal liver metastases: POCHER trial. $\mathrm{Br} J$ Cancer 103: 1542-1547.

25 Rees M, Tekkis PP, Welsh FK, O'Rourke T, John TG (2008) Evaluation of long-term survival after hepatic resection for metastatic colorectal cancer: a multifactorial model of 929 patients. Ann Surg 247: 125-135.

26 Ayez N, Lalmahomed ZS, Eggermont AM, ljzermans JN, de Jonge J, et al. (2012) Outcome of microscopic incomplete resection (R1) of colorectal liver metastases in the era of neoadjuvant chemotherapy. Ann Surg Oncol 19: 1618-1627.

27 Nuzzo G, Giuliante F, Ardito F, Vellone M, Giovannini I, et al. (2008) Influence of surgical margin on type of recurrence after liver resection for colorectal metastases: a single-center experience. Surgery 143: 384-393.

28 Karoui M, Koubaa W, Delbaldo C, Charachon A, Laurent A, et al. (2008) Chemotherapy has also an effect on primary tumor in colon carcinoma. Ann Surg Oncol 15: 3440-3446.

29 Matsui S, Osada S, Tomita H, Komori S, Mori R, et al. (2010) Clinical significance of aggressive hepatectomy for colorectal liver metastasis, evaluated from the HGF/c-Met pathway. Int J Oncol 37: 289-297.

30 Bates RC, Mercurio AM (2005) The epithelial-mesenchymal transition (EMT) and colorectal cancer progression. Cancer Biol Ther 4: 365-370. 
31 Chang HY, Kao MC, Way TD, Ho CT, Fu E (2011) Diosgenin suppresses hepatocyte growth factor (HGF)-induced epithelial-mesenchymal transition by down-regulation of Mdm2 and vimentin. J Agric Food Chem 59: 5357-5363.

32 Cañadas I, Taus A, González I, Villanueva X, Gimeno J, et al. (2014) High circulating hepatocyte growth factor levels associate with epithelial to mesenchymal transition and poor outcome in small cell lung cancer patients. Oncotarget 5: 5246-5256.

33 Ogunwobi OO, Liu C (2011) Hepatocyte growth factor upregulation promotes carcinogenesis and epithelial-mesenchymal transition in hepatocellular carcinoma via Akt and COX-2 pathways. Clin Exp Metastasis 28: 721-731.

34 Nagai T, Arao T, Furuta K (2011) Sorafenib inhibits the hepatocyte growth factor-mediated epithelial mesenchymal transition in hepatocellular carcinoma. Mol Cancer Ther 10: 169-177.

35 Tanahashi T, Osada S, Yamada A, Kato J, Yawata K, et al. (2013) Extracellular Signal-Regulated Kinase and Akt Activation Play a Critical Role in the Process of Hepatocyte Growth Factor-Induced Epithelial-Mesenchymal Transition. Int. J Oncol 42: 556-564..

36 Mani SA, Guo W, Liao MJ, Eaton EN, Ayyanan A, et al. (2008) The epithelial-mesenchymal transition generates cells with properties of stem cells. Cell 133: 704-715.

37 Singh A, Settleman J (2010) EMT, cancer stem cells and drug resistance: an emerging axis of evil in the war on cancer. Oncogene 29: 4741-4751.

38 Sabbah M, Emami S, Redeuilh G, Julien S, Prévost G, et al. (2008) Molecular signature and therapeutic perspective of the epithelialto-mesenchymal transitions in epithelial cancers. Drug Resist Updat 11: 123-151.

39 Wang Z, Li Y, Ahmad A, Azmi AS, Kong D, et al. (2010) Targeting miRNAs involved in cancer stem cell and EMT regulation: An emerging concept in overcoming drug resistance. Drug Resist Updat 13: $109-118$

40 Kajiyama H, Shibata K, Terauchi M (2007) Chemoresistance to paclitaxel induces epithelial-mesenchymal transition and enhances metastatic potential for epithelial ovarian carcinoma cells. Int J Onco 31: $277-283$

41 Rho JK, Choi YJ, Lee JK (2009) Epithelial to mesenchymal transition derived from repeated exposure to gefitinib determines the sensitivity to EGFR inhibitors in A549, a non-small cell lung cancer cell line. Lung Cancer 63: 219-226.

42 Yu SJ, Yu JK, Ge WT, Hu HG, Yuan Y, et al. (2011) SPARCL, Shp, MSH, E-cadherin, p, ADCY-2 and MAPK are prognosis-related in colorectal cancer. World J Gastroenterol 17: 2028-2036.

43 Koo JS, Jung W, Jeong J (2009) The predictive role of E-cadherin and androgen receptor on in vitro chemosensitivity in triple-negative breast Cancer. Jpn J Clin Oncol 39: 560-568.

44 Vinod BS, Antony J, Nair HH, Puliyappadamba VT, Saikia M, et al. (2013) Mechanistic evaluation of the signaling events regulating curcumin-mediated chemosensitization of breast cancer cells to 5-fluorouracil. Cell Death Dis 4: e505.

45 Sameshima S, Tomozawa S, Kojima M, Koketsu S, Motegi K, et al. (2008) 5-Fluorouracil-related gene expression in primary sites and hepatic metastases of colorectal carcinomas. Anticancer Res 28: 1477-1481.
46 Kumamoto K, Kuwabara K, Tajima Y (2014) Thymidylate synthase and thymidine phosphorylase mRNA expression in primary lesions using laser capture microdissection is useful for prediction of the efficacy of FOLFOX treatment in colorectal cancer patients with liver metastasis. Oncol Lett 3: 983-989.

47 Fukuta K, Osada S, Tanahashi T, Matsui S, Sasaki Y, et al. Increase in Chemotherapeutic Effect by Hepatocyte Growth Factor-Induced Epithelial-Mesenchymal Transition. Cancer in press.

48 Levina V, Su Y, Nolen B, Liu X, Gordin Y, et al. (2008) Chemotherapeutic drugs and human tumor cells cytokine network. Int J Cancer 123: 2031-2040.

49 Kemeny N (2007) Presurgical chemotherapy in patients being considered for liver resection. Oncologist 12: 825-839.

50 Nakano H, Oussoultzoglou E, Rosso E, Casnedi S, Chenard-Neu $\mathrm{MP}$, et al. (2008) Sinusoidal injury increases morbidity after major hepatectomy in patients with colorectal liver metastases receiving preoperative chemotherapy. Ann Surg 247: 118-124.

51 de Gramont A, Figer A, Seymour M, Homerin M, Hmissi A, et al. (2000) Leucovorin and fluorouracil with or without oxaliplatin as first-line treatment in advanced colorectal cancer. J Clin Oncol 18: 2938-2947.

52 Tournigand C, André T, Achille E, Lledo G, Flesh M, et al. (2004) FOLFIRI followed by FOLFOX6 or the reverse sequence in advanced colorectal cancer: a randomized GERCOR study. J Clin Oncol 22: 229237.

53 White RR, Schwartz LH, Munoz JA, Raggio G, Jarnagin WR, et al. (2008) Assessing the optimal duration of chemotherapy in patients with colorectal liver metastases. J Surg Oncol 97: 601-604.

54 Sasaki Y, Osada S, Mori R, Imai H, Tanaka Y, et al. (2013) Determining timing of hepatectomy for colorectal cancer with distant metastasis according to imaging-based tumor shrinkage ratio. Int J Med Sci 10: 1231-1241.

55 Adam R, Pascal G, Castaing D, Azoulay D, Delvart V, et al. (2004) Tumor progression while on chemotherapy: a contraindication to liver resection for multiple colorectal metastases? Ann Surg 240: 1052-1061.

56 Chiappa A, Bertani E, Makuuchi M, Zbar AP, Contino G, et al. (2009) Neoadjuvant chemotherapy followed by hepatectomy for primarily resectable colorectal cancer liver metastases. Hepatogastroenterology 56: 829-834.

57 Sasaki Y, Osada S, Imai H, Tanaka Y, Okumura N, et al. (2015) Tumor shrinkage direction of colorectal cancer liver metastases by chemotherapy. AntiCaner Res 35: 5485-5490.

58 Nakanishi M, Kuriu Y, Murayama Y, Konishi H, Komatsu S, et al. (2014) Efficacy of perioperative chemotherapy in patients with colorectal cancer undergoing hepatectomy for resectable synchronous liver metastases. Hepatogastroenterol 61: 1582-1587.

59 Folprecht G, Gruenberger T, Bechstein WO, Raab HR, Lordick F, et al. (2010) Tumour response and secondary resectability of colorectal liver metastases following neoadjuvant chemotherapy with cetuximab: the CELIM randomised phase 2 trial. Lancet Oncol 11: 38-47. 